\title{
Robust estimates of hydrogen-impact ionization cross sections over a wide energy range
}

\author{
A. F. Barghouty \\ California Institute of Technology, MC 220-47, Pasadena, California 91125
}

(Received 26 October 1999; published 29 March 2000)

\begin{abstract}
We describe a simple and effective procedure to estimate hydrogen-impact ionization cross sections over the energy range of tens of $\mathrm{keV} /$ nucleon to tens of $\mathrm{MeV} /$ nucleon. The procedure is valid in the first Born approximation using known or estimated electron-impact cross sections. The original Bates-Griffing relation between the two sets of cross sections is reformulated and a correction factor due to multiple transitions is introduced. Sample calculated ionization cross sections for helium and carbon collisions with hydrogen are presented.
\end{abstract}

PACS number(s): 34.10.+x, 34.70.+e, 52.20.-j

\section{INTRODUCTION}

Hydrogen-impact ionization cross sections for light ions in the energy range of $\sim \mathrm{keV} /$ nucleon to a few hundreds of $\mathrm{MeV} /$ nucleon are poorly known for many reactions of interest to astrophysics and plasma physics. Such cross sections and the corresponding ionization rates are critically needed in, e.g., transport models of energetic ions in space and laboratory plasmas.

For the energy range relevant to these studies the first Born approximation can be used to estimate the cross section $\sigma_{p}$ for the ion-hydrogen electron-loss process (e.g., [1]):

$$
X^{q}+H \rightarrow X^{q+1}+H^{*}+e^{\prime},
$$

if the ion's kinetic energy is above a certain threshold energy $E_{B}$, which is ionization-potential dependent. In process (1), $H^{*}$ denotes all bound and continuum states of $H$, but without specifying the states, while $e^{\prime}$ denotes the ejected electron, which can emerge with some kinetic energy imparted to it. The same approximation can also be applied for $\sigma_{e}$ from the electron-impact process

$$
X^{q}+e \rightarrow X^{q+1}+e+e^{\prime} .
$$

The original Bates-Griffing relation [2] is a general, but open, functional between the two sets of cross sections for the above two processes. For the same incident relative velocity and at high enough energies (i.e., the incident kinetic energy $\gg E_{B}$ ), the two cross sections approach a single value. For energies below this asymptotic region, which, for example, are more relevant to space plasma transport studies, the Bates-Griffing relation gives $\sigma_{e}$ in terms of $\sigma_{p}$ as a functional since the incident kinetic energies do not directly correspond to each other. The functional is not closed in the sense that $\sigma_{p}$ is evaluated at two different enregy points in order to estimate $\sigma_{e}$ at a single energy point that corresponds to the same incident velocity.

The availability of $E_{B}$-dependent, parametric estimates for $\sigma_{e}$ (e.g., [3-5]) makes it possible to estimate $\sigma_{p}$ using a relation like the Bates-Griffing. To that end, we will express $\sigma_{p}$ as a closed functional of $\sigma_{e}$ using the Bates-Griffing relation in the first Born approximation. In addition, a correction factor associated with multiple transitions, i.e., sum over shells, is introduced. The procedure is only meant to give a crude, but robust, first-order approximation to needed cross sections that otherwise either have not been measured or are difficult to calcualte theoretically. Sample applications to the direct-impact ionization of helium and carbon in collisions with hydrogen along with some available data are presented.

An implementation of this formalism in $\mathrm{F} 77 / 90$ and $\mathrm{C} / \mathrm{C}++$ portable routines along with the needed shells' data files (for elements up to $\mathrm{Fe}$ ) are available via anonymous FTP from thor.srl.caltech.edu/pub/ace/atomic. Implementation for other elements where estimates (or data) for $\sigma_{e}$ are available is straightforward.

\section{THE BATES-GRIFFING RELATION}

In the first Born approximation the collision is approximated as a transition from continuum to continuum states. In the center-of-mass frame the initial and final wave functions become pure momentum states of a single particle, and the transition matrix is essentially determined by a Coulombic potential and the amount of momentum transfer in the collision. For processes (1) and (2), ignoring any energy imparted to the ejected electron, the Born cross section is written as

$$
\sigma_{B}\left(E_{e}\right)=\frac{8}{\pi E_{e}} \int_{k_{\min }}^{k_{\max }} d k k^{-3}\left|\mathcal{M}_{f i}\right|^{2},
$$

where $E_{e}$ is the electron's incident kinetic energy, $k=k_{i}$ $-k_{f}$ is the relative momentum, and $k_{i}$ and $k_{f}$ are the initial and final relative momenta. The limits $k_{\min }$ and $k_{\max }$ are determined from energy conservation, and are dependent on the incident particle. Kinematically, the two cross sections $\sigma_{e}$ and $\sigma_{p}$ are different because these limits are different. For a single transition with ionization potential $V_{j}$, for protons ${ }^{1}$ the formal limits [6] are

$$
k_{\min }=\frac{V_{j}}{2 k_{i}}\left(1+\frac{1}{4} \frac{m_{e}}{\mu} \frac{V_{j}}{E_{e}}\right), \quad k_{\max } \rightarrow \infty,
$$

where $m_{e}$ is the electron's rest mass and $\mu$ the reduced mass of the ion-hydrogen system. For electrons the limits become

\footnotetext{
${ }^{1} \mathrm{We}$ treat atomic hydrogen as a proton because the correction factor (in $\mathcal{M}_{f i}$ ) due to the bound electron behaves like $k^{-4}$ in Eq. (3), which is negligible for the energy range of interest here.
} 


$$
k_{\min }=k_{i}-k_{i}\left(1-\frac{V_{j}}{E_{e}}\right)^{1 / 2}, \quad k_{\max }=k_{i}+k_{i}\left(1+\frac{V_{j}}{E_{e}}\right)^{1 / 2} .
$$

The matrix elements $\mathcal{M}_{f i}$ need not be evaluated explicitly. Instead, Eq. (3) for protons can be written as

$$
\sigma_{p}\left(E_{p} ; k\right)=\frac{\mu}{m_{e}} \frac{\mathcal{W}(k)}{E_{p}}
$$

where $E_{p}$ is the proton's incident kinetic energy such that $k_{\min }$ is the same in both the ion-electron and ion-proton systems. The function $\mathcal{W}$, whose exact form is not required, is defined as

$$
\frac{d \mathcal{W}(k)}{d k} \propto-k^{-3}\left|\mathcal{M}_{f i}\right|^{2}
$$

with the property that in the limit $k \rightarrow \infty, \mathcal{W} \rightarrow 0$. Similarly, for electrons the Born cross section becomes

$$
\sigma_{e}\left(E_{e} ; k\right)=\frac{\mathcal{W}(k)-\mathcal{W}\left(k^{\prime}\right)}{E_{e}},
$$

where $k^{\prime}=V_{j} / k$.

Now, defining $E_{B}$ as $\mu V_{j} / 4 m_{e}$ and $\gamma$ as $\left(1+E_{B} / E_{p}\right)^{2}$, and realizing that $E_{e}(k)=\left(\gamma m_{e} / \mu\right) E_{p}(k)$, the Bates-Griffing relation for $\sigma_{e}$ in terms of $\sigma_{p}$ can be written as

$$
\sigma_{e}\left(E_{e} ; k\right)=\gamma^{-1}\left(\sigma_{p}\left(E_{p} ; k\right)-\frac{E_{p}^{\prime}}{E_{p}} \sigma_{p}\left(E_{p}^{\prime} ; k^{\prime}\right)\right),
$$

with $E_{p}^{\prime}$ defined as $E_{B}^{2} / E_{p}$. In Eq. (9) $\sigma_{p}$ needs to be evaluated at two different energy points, $E_{p}$ and $E_{p}^{\prime}$, corresponding to two different relative momenta, $k$ and $k^{\prime}$, in order to estimate $\sigma_{e}$ at the energy point $E_{e}$. As such the BatesGriffing relation for $\sigma_{e}$ in terms of $\sigma_{p}$ is not a closed one. Next, we show how one can, to first order in $\left(k-k^{\prime}\right)$, reexpress the Bates-Griffing relation for $\sigma_{p}$ in terms of $\sigma_{e}$ at the same $k$.

\section{EXPRESSING $\sigma_{p}$ IN TERMS OF $\sigma_{e}$}

To express $\sigma_{p}$ as a closed functional of $\sigma_{e}$, we Taylor expand $\sigma_{p}\left(E_{p} ; k\right)$ about $\sigma_{p}\left(E_{p}^{\prime} ; k^{\prime}\right)$ to first order in $(k$ $\left.-k^{\prime}\right)$ as

$$
\sigma_{p}\left(E_{p} ; k\right)=\sigma_{p}\left(E_{p}^{\prime} ; k^{\prime}\right)+\left(k-k^{\prime}\right) \frac{d \sigma_{p}\left(E_{p} ; k\right)}{d k} .
$$

From Eqs. (6) and (8), to first order in $\left(k-k^{\prime}\right)$ with $\mu^{\prime}$ $=\mu / m_{e}$, we have

$$
\begin{gathered}
\frac{d \sigma_{p}\left(E_{p} ; k\right)}{d k}=\frac{\mu^{\prime}}{E_{p}} \frac{d \mathcal{W}}{d k}+\mu^{\prime} \mathcal{W} \frac{d E_{p}^{-1}}{d k}, \\
\frac{d \mathcal{W}}{d k}=\frac{\mathcal{W}(k)-\mathcal{W}\left(k^{\prime}\right)}{k-k^{\prime}}=\frac{E_{e}}{k-k^{\prime}} \sigma_{e}\left(E_{e} ; k\right) .
\end{gathered}
$$

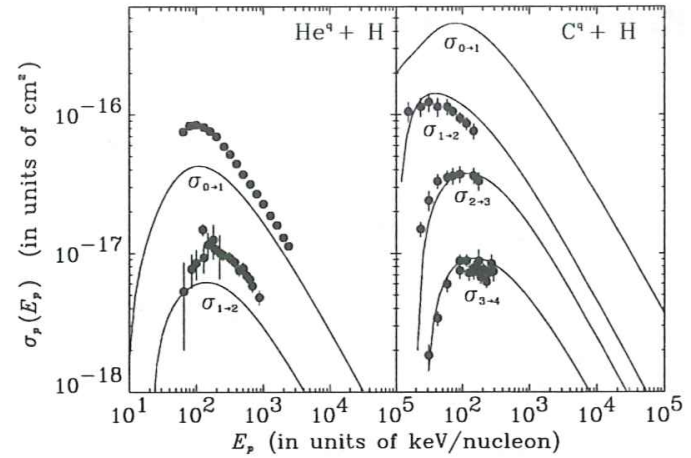

FIG. 1. Estimated hydrogen-impact ionization cross sections for helium and carbon. Curves depict the cross sections $\sigma_{0 \rightarrow 1}$ (i.e., the differential cross section for the reaction $X^{0}+H \rightarrow X^{1}+H^{*}+e^{\prime}$ ), $\sigma_{1 \rightarrow 2}$, etc., and terminate as the kinetic energy $E_{p} \rightarrow E_{B}^{+}$. Data for helium are from Refs. [7-10], and for carbon from Refs. [10,11].

Upon substituting Eqs. (11) and (12) in Eq. (10) and equating terms with Eq. (9), we get

$$
\sigma_{p}\left(E_{p}^{\prime} ; k^{\prime}\right)=-\frac{k-k^{\prime}}{1-\epsilon^{2}} \mu^{\prime} \mathcal{W} \frac{d E_{p}^{-1}}{d k}=\frac{2}{1+\epsilon} \sigma_{p}\left(E_{p} ; k\right),
$$

where $\epsilon=E_{B} / E_{p}$ and $E_{p}=1 / 4 \mu^{\prime} k^{2}$. Eliminating $\sigma_{p}\left(E_{p}^{\prime} ; k^{\prime}\right)$ in Eq. (9) and solving for $\sigma_{p}\left(E_{p} ; k\right)$ gives

$$
\sigma_{p}\left(E_{p} ; k\right)=\gamma\left(1-\frac{2 \epsilon^{2}}{1+\epsilon}\right)^{-1} \sigma_{e}\left(E_{e} ; k\right)
$$

which is a closed relation for $\sigma_{p}$ in terms of $\sigma_{e}$ at the same $k$.

Finally, since the Arnaud and Rothenflug [4,5] estimates we use for $\sigma_{e}$ are shell dependent and the above relation for $\sigma_{p}$ depends on each shell parametrically, the total, i.e., sum over shells, cross section becomes

$$
\sigma_{p}\left(E_{p} ; k\right)=\frac{1}{2}\left(1+N^{2}\right) \sum_{j=1}^{N} \sigma_{p}^{j}\left(E_{p} ; k\right) / j^{2}
$$

where $N$ is the number of shells and $\sigma_{p}^{j}\left(E_{p} ; k\right)$ is given by Eq. (14) for each ionization potential $V_{j}$. The factor $1 / 2 j^{2}$ assumes hydrogenlike degeneracy and $\left(1+N^{2}\right)$ is a normalization factor.

To illustrate, in Fig. 1 we show calculated ionization cross sections for helium and carbon where some available data are also shown.

\section{ACKNOWLEDGMENTS}

This work has been supported by NSF Grant No. 9810653 and NASA-JOVE Grant No. NAG8-1208. 
[1] M. R. C. McDowell and J. P. Coleman, Introduction to the Theory of Ion-Atom Collisions (North-Holland, Amsterdam, 1970).

[2] D. R. Bates and G. Griffing, Proc. Phys. Soc. London 66, 961 (1953)

[3] V. P. Shevelko et al., Mon. Not. R. Astron. Soc. 203, 45P (1983).

[4] M. Arnaud and R. Rothenflug, Astron. Astrophys. Suppl. Ser. 60, 425 (1985).

[5] M. Arnaud and J. Raymond, Astrophys. J. 398, 394 (1992).
[6] G. Peach, Proc. Phys. Soc. London 85, 709 (1965).

[7] J. M. Peart, K. Rinn, and K. T. Dolder, J. Phys. B 16, 1461 (1983).

[8] M. B. Shah and H. B. Gilbody, J. Phys. B 18, 899 (1985).

[9] M. F. Watts, K. F. Dunn, and H. B. Gilbody, J. Phys. B 19, L355 (1986).

[10] M. M. Sant'Anna et al., Phys. Rev. A 58, 1204 (1998).

[11] T. V. Goffe, M. B. Shah, and H. B. Gilbody, J. Phys. B 12, 3763 (1979). 
